\title{
Recurrence of atypical fibroxanthoma. Diagnosis and treatment
}

\author{
Leandro Ricardo Mattiola', Lyzandro Mattiola², Giovani Mattiola, Sandra Zucchi de Moraes Mattiolat, Carlos Eduardo Moura4, \\ Alexandre Kirschnick.
}

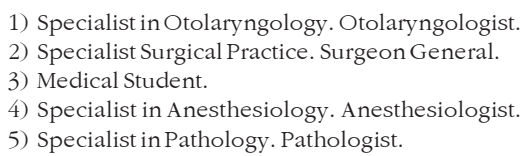

\section{SUMMARY}

Introduction: The soft tissue sarcomas (SPM) accounts for only $1 \%$ of malignant tumors of the adult population. The SPM is the most frequent malignant fibrous histiocytoma (MFH) that exhibits behavior characterized by the tendency to invasion of adjacent tissue and metastatic spread early. One of its variants is the atypical fibroxanthoma (FA).

Objective: To describe a case of probable recurrence of AF underwent surgical treatment and presentation of a literature review. Case report: Patient female, 63 years, presenting with a mass in the face about four inches and a history of prior resection of the lesion in the same topography. The patient underwent surgical resection with a diagnosis of AF.

Conclusion: The FA is a rare tumor histological diagnosis difficult. The correct histological diagnosis and patient follow-up are essential.

Keywords: sarcoma; malignant fibrous histiocytoma; neoplasms of soft tissue neoplasms ENT.

\section{INTRODUCTION}

Of the malignant tumors of the adult population only $1 \%$ are soft tissue sarcomas (SPM) (1). Of these, 5 to $16 \%$ occur in the head and neck (2.3). The malignant fibrous histiocytoma $(\mathrm{MFH})$ is a rare tumor and there are few cases described in the literature, and thus difficult to define prognosis and treatment 4 . It was described in the 60 s as a distinct type of SPM, and has been the most common histological type diagnosed since. The atypical fibroxanthoma (FA) is one of its variants. The SPM exhibit aggressive behavior, tendency to invasion and metastatic spread early. We present a case of probable recurrence of AF after two years, surgical treatment and a brief review of the literature.

\section{Case Report}

Female patient, 63 years old, came to us complaining of swelling in the face (cheek), left, with six months of evolution and rapid growth in the past two months. She reported no other symptoms and disorders. However, reported history of resection of a skin lesion on the same location for about two years. Whose histopathology was inconclusive on histological lineage, however, assured clear margins and no additional study was conducted for a conclusive diagnosis.

Physical examination revealed subcutaneous swelling in the left side of fibro-elastic consistency, adjusting, measuring approximately four by two centimeters, movable with respect to the depth and apparently fixed to the overlying skin, where scar visualized approximately three inches, corresponding prior to surgery. He had no palpable cervical lymph nodes. Computed tomography (CT) revealed an increase in volume in the subcutaneous tissue of the left face, with contrast medium uptake and cleavage plane with the left parotid gland deeply. We performed fine needle aspiration biopsy that showed only atypical cells. During this period there was an increase in lesion volume and apparent infiltrating the epidermis.

Due to suspicion of malignancy with rapid evolution, we opted for surgical resection of the lesion accompanied by frozen section intraoperative examination. This has not been able to define the histological lineage, but confirmed 
that it was invasive malignancy. Resection was performed with safety margins and included the overlying skin. Primary closure was performed and postoperatively the patient developed salivary fistula, treated with pressure dressings and healing after 15 days.

Pathology showed undifferentiated malignant neoplasm characterized by proliferation of spindle cells, pleomorphic epithelioid and some involving the dermis and subcutaneous tissue. Observing occasional mitotic figures. The surgical margins were free. The immunohistochemical study revealed for CD10 expression, favoring the diagnosis of atypical fibroxanthoma (malignant fibrous histiocytoma surface). The tumor was staged as NO M0 pT1a - stage IIB (UICC 2006). It was chosen to carry out radiotherapy or chemotherapy. The patient had no signs of local recurrence or distant metastasis when, after four months was detected on clinical examination new tumor in the topography of the mandibular body left in an area that had no contact with the resected lesion. A biopsy of the tumor showed that it was FA. The patient was referred to a tertiary hospital to give the sequel treatment, since it required at this time a more extensive resection followed by reconstruction of the mandible. Which was done so as radiotherapy.

\section{DisCUSSION}

The SPM represent only $1 \%$ of all cancers of the head and neck (5). They are grouped together because of similarities in their appearance pathological, clinical presentation and natural history, although they have different cellular origins (3.6). MFH is a rare tumor, but it can be in the region of the head and neck should be part of the differential diagnosis of primary cervical lesions. One of its variants is the FA. The clinical behavior is characterized by the tendency to invasion of adjacent soft tissues and early metastatic dissemination (7). Occasionally these tumors are associated with genetic syndromes or prior radiation. Most often there is no clear etiology (2).

Men are more affected than women (3:2). The most common presentation is as painless swelling (5). Many symptoms may occur depending on the location of the tumor (2). Physical examination usually reveals sub mucosal or subcutaneous tumor with distortion or destruction of adjacent structures. CT and magnetic resonance imaging (MRI) scans are the choice in most cases and provide information about bone involvement, extension and lymph node metastasis in addition to size and location (2). These tests can be used in a complementary way, especially in surgical planning (8). However, for a histological diagnosis in addition to the microscopic characteristics, it is necessary to perform immunohistochemical analysis (9).

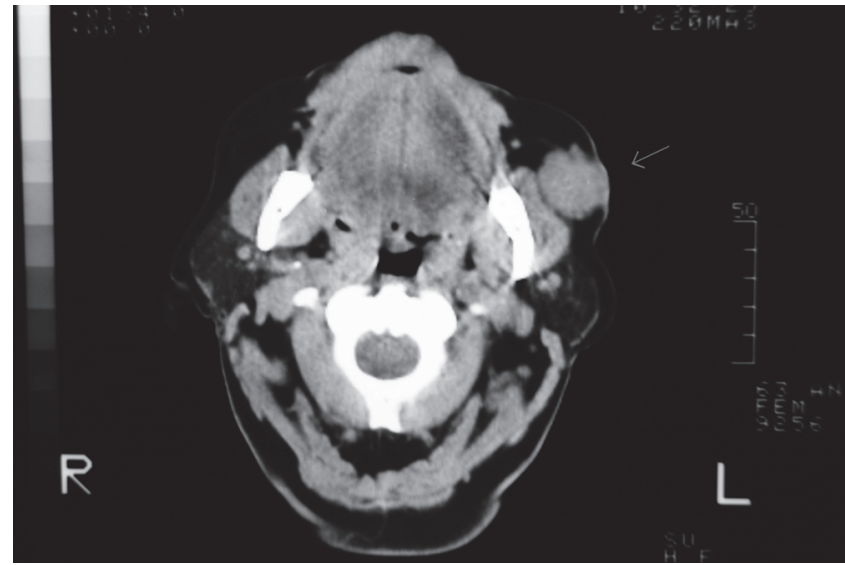

Figure 1. Tumor in the subcutaneous tissue of the left cheek. It is observed cleavage plane with glula to deep left.

The prognostic factors are histology grade, location, size and lymph node involvement (7). MFH is generally considered a lesion of high-grade malignancy $(10,11)$. Regarding location in the head and neck, is considered a worse prognosis with higher recurrence rates and lower disease-specific survival (12). Tumors larger than three centimeters in diameter negatively affect the prognosis. Literature data show that surgical safety margin is among the most important prognostic factors affecting local control, although its significance as a determinant of survival remains uncertain (13). In the study of Belal et al. (3), positive microscopic margins were associated with increased local recurrence. This author also found a significant correlation between adequate margins and disease-free survival and overall survival. In the head and neck high rates of positive margins are reflective of the closeness of relationships between adjacent structures. Local recurrence occurs in approximately $20 \%$ of cases, but the biggest cause of death is distant metastasis. The survival rate is $40 \%$ in the largest series, ranging from 19 to $75 \%$ (5). The authors differ if the local recurrence itself is a cause of distant metastases and increased mortality (5.14).

The mainstay of treatment is wide surgical resection with clear margins (1.3). Some authors consider elective neck dissection is not necessary since regional metastases are uncommon. Postoperative radiotherapy is essential for banks affected (3). It is also recommended for unresectable tumors and margins slim. The role of adjuvant chemotherapy is controversial (3), its primary indication is for cases of metastatic disease (15).

In the case of $\mathrm{AF}$ patients had a facial tumor with no metastases and that probably it was a local recurrence of 
resected lesion two years ago, but without histopathological diagnosis. We opted for surgical treatment, associated with intraoperative frozen due to great suspicion of malignancy, diagnostic difficulty and rapid progression at the time of preoperative investigation. The intraoperative frozen was not able to define the histological diagnosis, as this can only be determined after immunohistochemistry. After the histological diagnosis of AF (HFM) neck dissection was not performed due to lack of commitment to regional clinical and radiological evaluations. Also we decided not to radiotherapy of believing in a small possibility of recurrence due to tumor staging and the wide margins of resection (minimum of $1 \mathrm{~cm}$ ) secured by microscopic analysis. The literature is unclear as to the minimum margin of safety. Chemotherapy was not used in view of the controversies reported in the literature. But after four months of surgery was the emergence of new tumor in the left side, which after biopsy showed that it was FA. The patient was referred to a tertiary hospital for treatment to follow.

\section{CONCLUSION}

The FA is a rare and difficult histological diagnosis. Surgery with wide resection of the lesion is the mainstay of treatment. The identification of tumor histology is of fundamental importance, as well as the postoperative follow-up. In this case the histological diagnosis of the primary lesion would provide better preoperative planning and early surgical intervention after local recurrence.

\section{REFERENCES}

1. Salo JC, Lewis JJ, Woodruff JM. Malignant fibrous histiocytoma of the extremity. Cancer, 1999;85:1765-72.

2. Sturgis EM, Potter BO, Sarcomas of the head and neck region. Curr Opin Oncol, 2003;15:239-52.

3. Belal A, Kandil A, Allam A, Khafaga Y, Husseiny G, ElEnbaby A, Memon M, Younge D, Moreau P, Gray A, Schultz A. Malignant fibrous histiocytoma a retrospective study of 109 cases. Am J Clin Oncol, 2002;25(1):16-22.

4. Barbosa FH, Rangel MO, Coelho SR. Patrocínio JA, Patrocínio LG. Histiocitoma fibroso maligno de laringe.
Arquivos Internacionais de Ororrinolaringologia, 2006, 103:242-244.

5. Huber GF, Matthews TW, Dort JC. Soft-tissue sarcomas of the head and neck: a retrospective analysis of the Alberta experience 1974 to 1999. Laryngoscope, 2006;116:780-85.

6. Fujimura T, Okuyama R, Terui T, Okuno K, Masu A, Masu T, Chiba S, Kunii T, Tagami H, Aiba S. Myxofibrosarcoma (myxoid malignant fibrous histiocytoma) showing cutaneous presentation: report of two cases. J Cutan Pathol, 2005;32:512-15.

7. Rosenberg SA, Glatestein EJ. Perspectives on the role of surgery and radiation therapy in the treatment of soft tissue sarcomas of the extremities. Semin Oncol, 1981;8:190-200.

8. Patel SC, Silbergleit R, Talati SJ. Sarcomas of the head and neck. Top Magn Reson Imaging, 1999;10:362-375.

9. Han M, Lee BJ, Jang YJ, Chung YS. Clinical value of officebased endoscopic incisional biopsy in diagnosis of nasal cavity masses. Otol Head \& Neck Surg, 2010;143:341-347.

10. Farhood AI, Hajdu SI, Shiu MH. Soft tissue sarcomas of the head and neck in adults. Am J Surg, 1990;160:365-69.

11. Lawrence W Jr. Operative management of soft tissue sarcomas: impact of anatomic site. Semin Surg Oncol, 1994;10:340-46.

12. LeVayJ, O'Sullivan B, Catton C. Outcome and prognostic factors in soft tissue sarcoma in the adult. Int J Radiat Oncol Biol Phys, 1993;27:1091-99.

13. Evans RA. Soft tissue sarcoma: the enigma of local recurrence. J Surg Oncol, 1993;53:88-91.

14. Pisters PW, Harisson LB, Leung DH. Long-term results of a prospective randomized trial of adjuvant brachytherapy in soft tissue sarcoma. J Clin Oncol, 1996;14:859-68.

15. Fell W, Housini I, Marple B. Radiation-induced malignant fibrous histiocytoma of the head and neck. Otolaryngol Head Neck Surg, 1998;118:527-529. 